\title{
Observation of the orbital circular photogalvanic effect
}

\author{
P. Olbrich, ${ }^{1}$ S. A. Tarasenko, ${ }^{2}$ C. Reitmaier, ${ }^{1}$ J. Karch, ${ }^{1}$ D. Plohmann,,${ }^{1}$ Z. D. Kvon, ${ }^{3}$ and S. D. Ganichev ${ }^{1}$ \\ ${ }^{1}$ Terahertz Center, University of Regensburg, 93040 Regensburg, Germany \\ ${ }^{2}$ A.F. Ioffe Physico-Technical Institute, Russian Academy of Sciences, 194021 St. Petersburg, Russia \\ ${ }^{3}$ Institute of Semiconductor Physics, Russian Academy of Sciences, 630090 Novosibirsk, Russia
}

(Received 26 January 2009; published 6 March 2009)

\begin{abstract}
We report on the observation of the circular photogalvanic effect in Si-metal-oxide-semiconductor fieldeffect transistors with inversion channel excited by terahertz radiation. We demonstrate that in spite of the fact that the photocurrent is caused by transfer of the photon angular momentum to free carriers, it is not due to spin orientation but has a pure orbital origin. It results from the quantum interference of different pathways contributing to the free-carrier absorption of monochromatic radiation.
\end{abstract}

DOI: 10.1103/PhysRevB.79.121302

PACS number(s): 78.40.Fy, 72.40.+w, 73.40.Qv, 78.20.-e

The spin of electrons and holes in solid-state systems is an intensively studied quantum mechanical property showing a large variety of interesting physical phenomena. One of the most frequently used and powerful methods of generation and investigation of spin polarization is optical orientation with circularly polarized light. ${ }^{1}$ Besides purely optical phenomena such as circularly polarized photoluminescence, the optical generation of an unbalanced spin distribution in a semiconductor may lead to helicity-dependent photocurrents, e.g., circular photogalvanic effect (CPGE) ${ }^{2-4}$ CPGE current is excited only by light of nonzero helicity and reverses its direction upon switching the sign of circular polarization. So far, the CPGE has only been detected in materials with strong spin-orbit coupling and described by microscopic mechanisms based on spin-related processes. ${ }^{2-4}$

Here we report on the observation of the CPGE caused by absorption of terahertz radiation in Si-metal-oxidesemiconductor field-effect transistors (Si-MOSFETs). The experimental demonstration of the existence of a helicitysensitive photocurrent in Si-based structures is of particular interest. Silicon is characterized by a vanishingly small spinorbit coupling which makes spin-related mechanisms of the CPGE ineffective and, therefore, cannot account for the observed helicity-dependent photocurrent. Thus, an access in explaining the CPGE is required, involving mechanisms of pure orbital (spin-unrelated) origin. Here, we show that the CPGE in our structures is due to quantum interference of different pathways contributing to monochromatic radiation absorption. This effect has been predicted theoretically ${ }^{5}$ (see also Refs. 6 and 7) but not observed so far. Quantum interference plays an important role in various transport and optical phenomena. It has also been shown that it can induce photocurrents. Here, however, in contrast to the well-known photocurrents caused by quantum interference of one- and two-photon absorption processes in two color light, ${ }^{8-11}$ the photocurrent is due to quantum interference in the elementary one-photon absorption process.

We study $n$-type MOSFETs prepared on miscut Si surfaces to reduce spacial symmetry and enable photocurrents at normal incidence. The surfaces of our samples are tilted by the angle $\theta=9.7^{\circ}$ (sample 1) or $\theta=10.7^{\circ}$ (sample 2) from the (001) plane around $x \|[1 \overline{1} 0]$. We note, that the point group describing the miscut transistors is $C_{s}$ with $x$ normal to the mirror reflection plane. Two transistors of $1.2 \times 0.4 \mathrm{~mm}^{2}$ size oriented along and normal to the inclination direction $\boldsymbol{A} \| y$ with semitransparent $\mathrm{Ti}$ gates of $10 \mathrm{~nm}$ thickness are prepared on each substrate. Variation in the gate voltage $V_{g}$ from 1 to $10 \mathrm{~V}$ enables us to change the carrier density $N_{s}$ from 1.5 to $15 \times 10^{11} \mathrm{~cm}^{-2}$ and the energy spacing $\varepsilon_{21}$ between the size-quantized subbands $e 1$ and $e 2$ from 2 to 20 meV. The peak electron mobility $\mu$ in the channel is about $10^{3}$ and $2 \times 10^{4} \mathrm{~cm}^{2} / \mathrm{V} \mathrm{s}$ at $T=296$ and $4.2 \mathrm{~K}$. For optical excitation we used a pulsed $\mathrm{NH}_{3}$ laser. ${ }^{3}$ The laser generates radiation pulses $(\approx 100 \mathrm{~ns})$ with a power $P \simeq 5 \mathrm{~kW}$ and wavelengths $\lambda=76,90.5$, and $148 \mu \mathrm{m}$ corresponding to the photon energies $\hbar \omega=16.3,13.7$, and $8.4 \mathrm{meV}$, respectively. Applying $\lambda / 4$ plates we modify the radiation helicity as $P_{\text {circ }}=\sin 2 \varphi$, where $\varphi$ is the angle between the plate optical axis and the incoming laser polarization. The photocurrents are measured between source and drain via the voltage drop across a $50 \Omega$ resistor.

Irradiating MOSFET structures at normal incidence (see inset to Fig. 1) we observed a photocurrent signal with the temporal structure reproducing the laser pulse. The photocurrent detected by the transistors aligned perpendicular to $\boldsymbol{A}$ reverses its direction upon switching the radiation helicity from left- to right-handed circular polarizations and is caused by the CPGE. In contrast, the photocurrent measured in transistors aligned along $\boldsymbol{A}$ is the same for both polarizations and

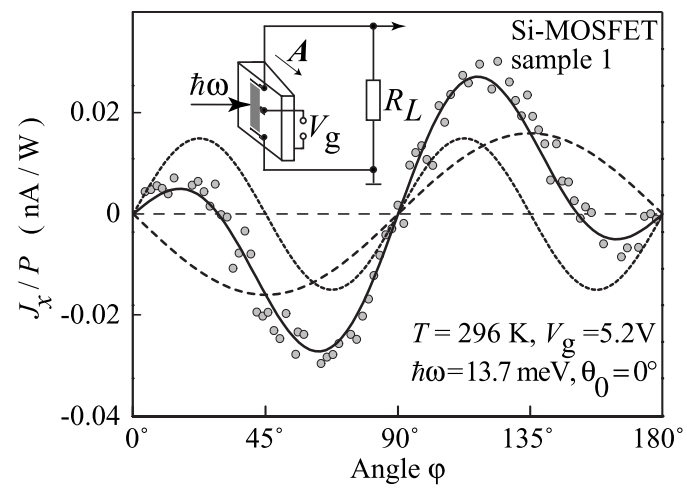

FIG. 1. Normalized photocurrent $J_{x} / P$ measured in sample 1 as a function of the angle $\varphi$. Full line is a fit to Eq. (1). Dashed and dotted lines show the CPGE and LPGE contributions, respectively. The inset sketches the experimental setup. 


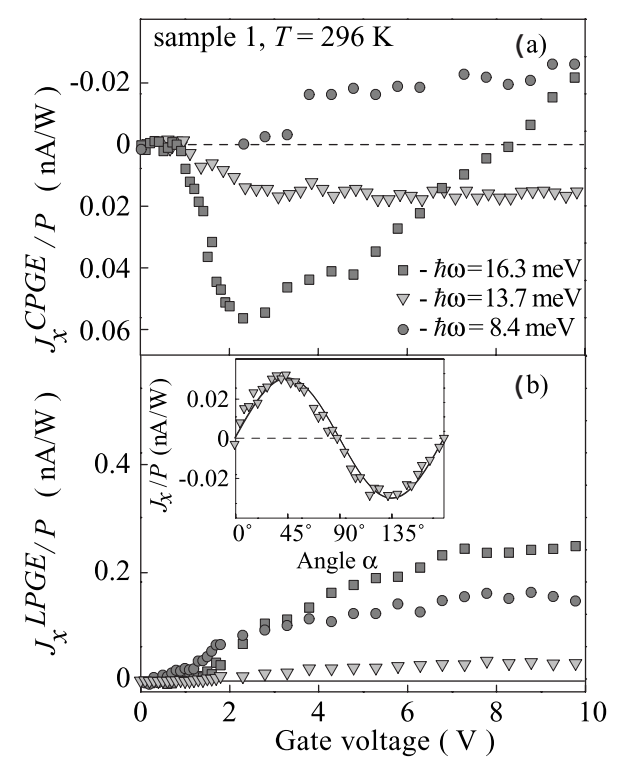

FIG. 2. Normalized photogalvanic currents as a function of the gate voltage. (a) CPGE and (b) LPGE. The inset shows the LPGE current as a function of the azimuth angle $\alpha$.

is due to linear photogalvanic effect (LPGE). These observations are in agreement with the phenomenological theory of the photogalvanic effects in structures of $C_{s}$ symmetry. ${ }^{4} \mathrm{Be}-$ low we focus only on transistors showing the CPGE.

First we discuss data obtained at room temperature. Figure 1 shows the dependence of the photocurrent on the angle $\varphi$ which can be well fitted by the phenomenological equation ${ }^{2,3}$

$$
J(\varphi)=J_{C} \sin 2 \varphi+\left(J_{L} / 2\right) \sin 4 \varphi .
$$

Here, the first and the second terms describe the CPGE and LPGE, respectively. Figure 1 shows the substantial contribution of the CPGE to the total current. This feature persists for all $V_{g}$ and $\hbar \omega$ used in our experiments.

Figure 2(a) shows the dependences of the CPGE contributions $J_{C}$ to the total photocurrent measured as a function of the gate voltage $V_{g}$. The CPGE contribution is obtained by taking the difference between photoresponses to the rightand left-handed radiations yielding the CPGE current $J_{C}$ $=\left[J\left(\varphi=45^{\circ}\right)-J\left(\varphi=135^{\circ}\right)\right] / 2$. Applying linearly polarized radiation which excludes CPGE we also investigated the LPGE. Using $\lambda / 2$ plates we varied the angle $\alpha$ between the electric field of radiation and the $x$ axis and obtained that the LPGE can be well fitted by the phenomenological equation ${ }^{3}$

$$
J(\alpha)=J_{L} \sin 2 \alpha,
$$

with the same parameter $J_{L}$ as used in Eq. (1). The dependence of the LPGE contribution on the gate voltage is shown in Fig. 2(b) where $J_{L}=\left[J\left(\alpha=45^{\circ}\right)-J\left(\alpha=135^{\circ}\right)\right] / 2$ is plotted for several radiation photon energies.

As follows from Fig. 2(b), the LPGE current has the same sign for every $\hbar \omega$ used and its magnitude increases with the gate voltage. Such a dependence can be attributed to the increase of the electron density in the inversion channel and, therefore, the Drude absorption enhancement. The CPGE be-

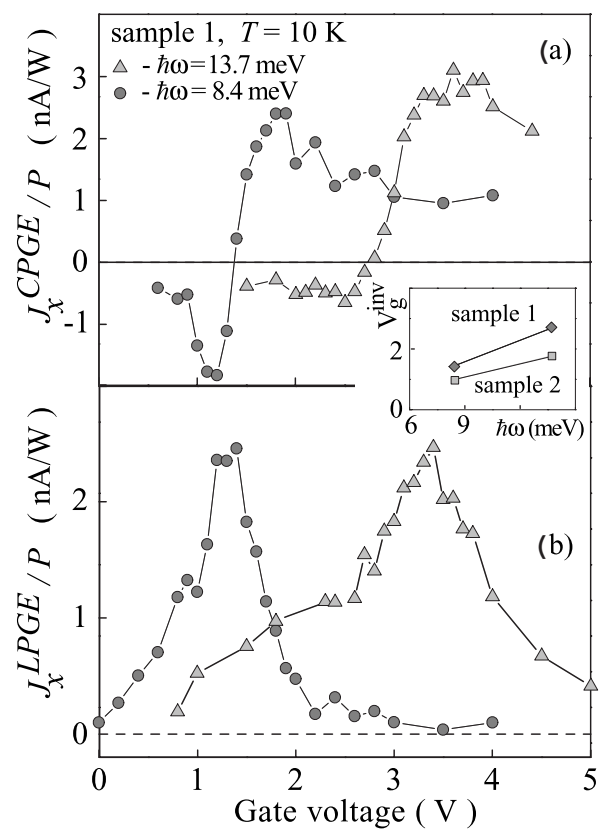

FIG. 3. (a) CPGE and (b) LPGE currents as a function of the $V_{g}$. The inset shows $V_{g}^{\text {inv }}$ as a function $\hbar \omega$.

havior, in contrast, is more complicated [see Fig. 2(a)]. We observe that the direction of the CPGE current is opposite for $\hbar \omega=8.4$ and $13.7 \mathrm{meV}$. Moreover, for $\hbar \omega=16.3 \mathrm{meV}$ the signal changes its sign with the $V_{g}$ increase. We note that we cannot attribute this gate voltage to any characteristic energy in the band structure.

At low temperatures the behavior of the photocurrent upon variation in the radiation polarization remains the same. All data can be well fitted by Eqs. (1) and (2) with comparable magnitudes of $J_{C}$ and $J_{L}$ proving the presence of both CPGE and LPGE. The gate voltage behavior, however, changes drastically as demonstrated in Fig. 3(a) for sample 1. The LPGE current, instead of smooth dependence observed at room temperature, shows a resonant response [see Fig. 3(b)]. The peak position depends on the photon energy and corresponds to the $\hbar \omega \cong \varepsilon_{21}$ as it has been additionally proved by the photoconductive measurements in biased transistors. The CPGE photocurrent changes its sign upon $V_{g}$ variation and vanishes at resonance, $\hbar \omega \cong \varepsilon_{21}$, where the LPGE achieves its maximum (see Fig. 3). This is in contrast to the room-temperature data where inversion has been detected for $\hbar \omega=16.3 \mathrm{meV}$ only and did not fit to the intersubband resonance. The inset of Fig. 3 shows the gate voltages of the CPGE inversion, $V_{g}^{\text {inv }}$, as a function of the photon energy.

The observation of the CPGE apart the intersubband resonance demonstrates that the free-carrier absorption of circularly polarized light gives rise to the helicity-dependent current. Below we consider theoretically this process and show that the CPGE is caused by the interference of different pathways contributing to the radiation absorption. Figure 4(a) sketches the indirect optical transitions within the ground subband $e 1$. Due to the energy and momentum conservation, the transitions can only occur if the electron-photon interaction is accompanied by simultaneous electron scattering by 

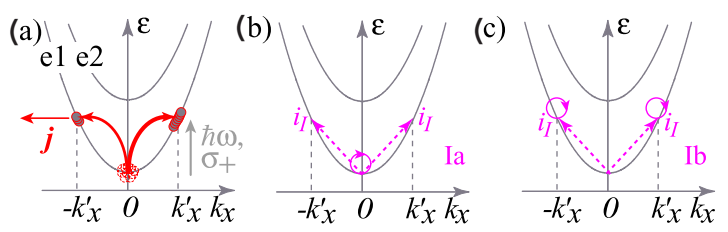

(d)

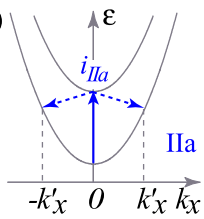

(e)

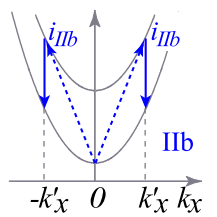

FIG. 4. (Color online) Microscopic model of the CPGE. (a) Indirect optical transitions due to absorption of circularly polarized light. Bend arrows of various thickness indicate the difference in transition rates caused by the quantum interference of various pathways. (b)-(e) Pathways via intermediate states $i_{\mathrm{I}}$ and $i_{\mathrm{II}}$. Here, the solid arrows illustrate electron-photon interaction and the dashed arrows show scattering events.

phonons or static defects. Such optical transitions are treated as second-order virtual processes via intermediate states. Figures 4(b)-4(d) show possible absorption pathways with the intermediate states in the $e 1$ and $e 2$ subbands.

The dominant contribution to the Drude absorption involves intermediate states within the $e 1$ subband. Such transitions (path I) are shown in Figs. 4(b) and 4(d) for the process where the electron-photon interaction is followed by electron scattering [Fig. 4(b)] and the inverted sequence process [Fig. 4(d)]. The matrix element of the intrasubband optical transitions with intermediate states in the $e 1$ subband on the vicinal silicon surface has the form ${ }^{7}$

$$
M_{\mathbf{k}^{\prime} \mathbf{k}}^{(\mathrm{I})}=\frac{e A}{c \omega}\left[\frac{\left(k_{x}^{\prime}-k_{x}\right) e_{x}}{m_{x x}}+\frac{\left(k_{y}^{\prime}-k_{y}\right) e_{y}}{m_{y y}}\right] V_{11},
$$

where $e$ is the electron charge, $A$ is the amplitude of the electromagnetic wave, $\omega$ is the radiation frequency, $m_{x x}$ $=m_{\perp}$ and $m_{y y}=\left(m_{\perp} \cos ^{2} \theta+m_{\|} \sin ^{2} \theta\right)$ are the effective electron masses in the channel plane being different from each other due to the deviation of the channel plane from (001) by the angle $\theta, m_{\|}$and $m_{\perp}$ are the longitudinal and transverse effective masses in the valley in bulk Si, $\boldsymbol{e}=\left(e_{x}, e_{y}\right)$ is the unit vector of the light polarization, and $V_{11}$ is the matrix element of electron scattering within the subband $e 1$. While the matrix element is odd in the wave vector, the absorption probability given by the squared matrix element is even in $\left(\boldsymbol{k}^{\prime}-\boldsymbol{k}\right)$. Thus, this type of processes alone does not introduce an asymmetry in the carrier distribution in $\boldsymbol{k}$ space and, consequently, does not yield a current.

Pathways II via states in the $e 2$ subband are sketched in Figs. 4(d) and 4(e). They involve virtual intersubband optical transitions which in miscut structures are allowed by selection rules even at normal incidence of light. ${ }^{6,12}$ The matrix element of path II optical transitions has the form

$$
M_{\mathbf{k}^{\prime} \mathbf{k}}^{(\mathrm{II})}=2 i \frac{e A}{c \hbar} \frac{m_{z z}}{m_{y z}} \frac{\hbar \omega \varepsilon_{21} z_{21}}{(\hbar \omega)^{2}-\varepsilon_{21}^{2}} e_{y} V_{12},
$$

where

$$
\begin{gathered}
1 / m_{z z}=\cos ^{2} \theta / m_{\|}+\sin ^{2} \theta / m_{\perp}, \\
1 / m_{y z}=\left(1 / m_{\perp}-1 / m_{\|}\right) \cos \theta \sin \theta
\end{gathered}
$$

is the off-diagonal component of the reciprocal effective mass tensor, $z_{21}$ is the coordinate matrix element, and $V_{12}$ is the matrix element of intersubband scattering. Equation (4) shows that this type of indirect transitions is independent of $\boldsymbol{k}$ and, consequently, also does not result in an electric current.

The photocurrent emerges due to the quantum interference of all virtual transitions considered above. Indeed, the total probability for the real optical transition $\boldsymbol{k} \rightarrow \boldsymbol{k}^{\prime}$ is given by the squared modulus of the sum of matrix elements describing individual pathways

$$
W_{\boldsymbol{k}^{\prime} \boldsymbol{k}} \propto\left|M_{\boldsymbol{k}^{\prime} \boldsymbol{k}}^{(\mathrm{I})}\right|^{2}+\left|M_{\boldsymbol{k}^{\prime} \boldsymbol{k}}^{(\mathrm{II})}\right|^{2}+2 \operatorname{Re}\left[M_{\boldsymbol{k}^{\prime} \boldsymbol{k}^{\prime}}^{(\mathrm{I})} M_{\boldsymbol{k}^{\prime} \boldsymbol{k}}^{(\mathrm{II}) *}\right] .
$$

Beside the probabilities of individual processes given by the first and the second term in the right-hand side of this equation, it contains the interference term. By using Eqs. (3) and (4) we derive for the latter term

$$
\operatorname{Re}\left[M_{\boldsymbol{k}^{\prime} \boldsymbol{k}}^{(\mathrm{I})} M_{\boldsymbol{k}^{\prime} \boldsymbol{k}}^{(\mathrm{II}) *}\right] \propto\left(k_{x}^{\prime}-k_{x}\right) i\left(e_{x} e_{y}^{*}-e_{x}^{*} e_{y}\right) F(\hbar \omega) .
$$

This term is linear in the wave vector and, therefore, results in different rates for the transitions to the positive and negative $k_{x}^{\prime}$. This, in turn, leads to an imbalance in the carrier distribution between $k_{x}^{\prime}$ and $-k_{x}^{\prime}$, i.e., to an electric current $j_{x}$. Such a difference in the real optical transition rates caused by constructive or destructive interference of various pathways is illustrated in Fig. 4(a). Moreover, the sign of the interference term is determined by the radiation helicity because $i\left(e_{x} e_{y}^{*}-e_{x}^{*} e_{y}\right)=\hat{o}_{z} P_{\text {circ }}$, where $\hat{\boldsymbol{o}}$ is a unit vector pointing along the light propagation direction. Therefore, the imbalance of the carrier distribution in $\boldsymbol{k}$ space and, consequently, the photocurrent reverse upon switching the light helicity.

Equation (6) also explains the inversion of the CPGE by varying the energy separation between the subbands from $\varepsilon_{21}<\hbar \omega$ to $\varepsilon_{21}>\hbar \omega$. Indeed, the interference term is proportional to the function $F(\hbar \omega) \propto 1 /\left[(\hbar \omega)^{2}-\varepsilon_{12}^{2}\right]$, which stems from the matrix element describing virtual transitions via the $e 2$ subband [see Eq. (4)]. In the vicinity of the absorption peak, the photocurrent increases drastically and undergoes spectral inversion. In real structures the dependence becomes smooth because of the broadening but the inversion remains. At room temperature the excited subbands, $e 2, e 3$, etc., are also occupied in the equilibrium and contribute to the current formation. Therefore the spectral behavior of the CPGE is more complicated and the point of inversion does not correspond to $\hbar \omega=\varepsilon_{21}$ anymore, as observed in experiment.

Assuming the electron scattering by short-range static defects, we write for the photocurrent ${ }^{5,7}$

$$
\begin{aligned}
\boldsymbol{j}= & e \frac{8 \pi}{\hbar} \sum_{\boldsymbol{k}, \boldsymbol{k}^{\prime}}\left[\tau_{p}\left(\varepsilon_{\boldsymbol{k}^{\prime}}\right) \boldsymbol{v}\left(\boldsymbol{k}^{\prime}\right)-\tau_{p}\left(\varepsilon_{\boldsymbol{k}}\right) \boldsymbol{v}(\boldsymbol{k})\right]\left[f\left(\varepsilon_{\boldsymbol{k}}\right)\right. \\
& \left.-f\left(\varepsilon_{\boldsymbol{k}^{\prime}}\right)\right] 2 \operatorname{Re}\left[M_{\boldsymbol{k}^{\prime} \boldsymbol{k}^{\mathrm{I}}}^{(\mathrm{I})} M_{\boldsymbol{k}^{\prime} \boldsymbol{k}}^{(\mathrm{II}) *}\right] \delta\left(\varepsilon_{\boldsymbol{k}^{\prime}}-\varepsilon_{\boldsymbol{k}}-\hbar \omega\right),
\end{aligned}
$$

where $\tau_{p}\left(\varepsilon_{k}\right)$ is the momentum relaxation time, $v_{x}(\boldsymbol{k})$ $=\hbar k_{x} / m_{x x}$ and $v_{y}(\boldsymbol{k})=\hbar k_{y} / m_{y y}$ are the velocity components, 
$\varepsilon_{\boldsymbol{k}}$ is the electron kinetic energy measured from the subband bottom, $f\left(\varepsilon_{k}\right)$ is the function of equilibrium carrier distribution in the subband $e 1$, and factor 8 in Eq. (7) accounts for the spin and valley degeneracy. Finally, we derive for the circular photocurrent density in Si-MOSFET structures with a small declination angle $\theta$

$$
j_{x}=e \tau_{p} \frac{m_{\|}}{m_{y z}} \frac{\left\langle V_{11} V_{12}\right\rangle}{\left\langle V_{11}^{2}\right\rangle} \frac{\varepsilon_{21} z_{21} \omega \eta_{x}}{\varepsilon_{21}^{2}-(\hbar \omega)^{2}} \hat{o}_{z} I P_{\text {circ }},
$$

where $\eta_{x}$ is the channel absorbance for the radiation polarized along the $x$ axis, $I$ is the radiation intensity, and the angle brackets stand for averaging over the spacial distribution of scatterers. Equation (8) describes the CPGE caused by the free-carrier absorption at $\hbar \omega<\varepsilon_{21}$ when the kinetic energy of photoexcited carriers is smaller than $\varepsilon_{21}$. For electrons generated with $\hbar \omega>\varepsilon_{21}$ the momentum relaxation time gets shorter because of the additional relaxation channel caused by the intersubband scattering. Consequently, the magnitude of the current is smaller for $\hbar \omega>\varepsilon_{21}$ (low $V_{g}$ ) than that at $\hbar \omega<\varepsilon_{21}$ (high $V_{g}$ ). This can be responsible for the observed asymmetry in the gate voltage dependence of the photocurrent in the intersubband resonant vicinity [see Fig. 3(a)]. The gate voltage changes also the channel profile which can be taken into account assuming that the ratio $\left\langle V_{11} V_{12}\right\rangle /\left\langle V_{11}^{2}\right\rangle$ and the coordinate matrix element $z_{21}$ depend on $V_{g}$. At $\hbar \omega \simeq \varepsilon_{21}$, possible contributions to the CPGE due to the intersubband optical transitions as well as the scattering-induced broadening of the absorption peak should also be taken into account. ${ }^{6}$

The magnitude of the CPGE detected in sample 1 for $\hbar \omega=8.4 \mathrm{meV}$ and $V_{g}=3 \mathrm{~V}$ is $J_{x} / P \sim 1 \mathrm{nA} / \mathrm{W}$, yielding the current density $j_{x} / I \sim 0.1 \mathrm{nA} \mathrm{cm} / \mathrm{W}$. The same order of magnitude is obtained from Eq. (8) for the structure with the vicinal angle $\theta=9.7^{\circ}$, the carrier density $N_{s}=5 \times 10^{11} \mathrm{~cm}^{-2}$ $\left(V_{g}=3 \mathrm{~V}\right)$, the channel width $a=80 \AA$, and the structure asymmetry degree $\left\langle V_{11} V_{12}\right\rangle /\left\langle V_{11}^{2}\right\rangle=10^{-2}$.

To summarize, we demonstrate in experiments on $\mathrm{Si}$ based structures that the photon helicity-dependent photocurrents can be generated in low-dimensional semiconductors even with vanishingly small spin-orbit interaction. The mechanism of the photocurrent formation is based on the quantum interference of different pathways contributing to the radiation absorption.

We thank E. L. Ivchenko, V. V. Bel'kov, L. E. Golub, W. Prettl, and S. N. Danilov. The financial support from the DFG (SFB 689) and the RFBF is gratefully acknowledged.
${ }^{1}$ Optical Orientation, edited by F. Meier, B. P. Zakharchenya (Elsevier Science, Amsterdam, 1984).

${ }^{2}$ E. L. Ivchenko, Optical Spectroscopy of Semiconductor Nanostructures (Alpha Science Int., Harrow, UK, 2005).

${ }^{3}$ S. D. Ganichev and W. Prettl, Intense Terahertz Excitation of Semiconductors (Oxford University Press, New York, 2006).

${ }^{4}$ E. L. Ivchenko and S. D. Ganichev, in Spin Physics in Semiconductors: Spin Photogalvanics, edited by M. I. D'yakonov (Springer, Berlin, 2008).

${ }^{5}$ S. A. Tarasenko, JETP Lett. 85, 182 (2007).

${ }^{6}$ L. I. Magarill and M. V. Entin, Sov. Phys. Solid State 31, 1299 (1989).
${ }^{7}$ E. L. Ivchenko and G. E. Pikus, Superlattices and Other Heterostructures (Springer-Verlag, Berlin, 1997).

${ }^{8}$ M. V. Entin, Sov. Phys. Semicond. 23, 664 (1989).

${ }^{9}$ A. Hache, Y. Kostoulas, R. Atanasov, J. L. P. Hughes, J. E. Sipe, and H. M. van Driel, Phys. Rev. Lett. 78, 306 (1997).

${ }^{10}$ R. D. R. Bhat and J. E. Sipe, Phys. Rev. Lett. 85, 5432 (2000).

${ }^{11}$ M. J. Stevens, A. L. Smirl, R. D. R. Bhat, J. E. Sipe, and H. M. van Driel, J. Appl. Phys. 91, 4382 (2002).

${ }^{12}$ G. M. Gusev, Z. D. Kvon, L. I. Magarill, A. M. Palkin, V. I. Sozinov, O. A. Shegai, and V. M. Entin, JETP Lett. 46, 33 (1987). 